Military Technical College

Kobry El-Kobbah,

Cairo, Egypt

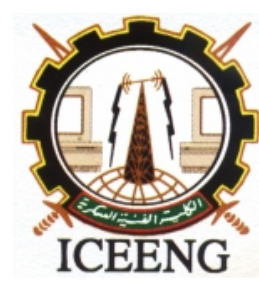

$7^{\text {th }}$ International Conference on Electrical Engineering ICEENG 2010

\title{
Development of new fuzzy logic-based ant colony optimization algorithm for combinatorial problems
}

By
Ahmed Rabie Ginidi *
Ahmed M. A. Kamel *
Hassen Taher Dorrah *

\section{$\underline{\text { Abstract: }}$}

This paper is directed towards developing a new fuzzy-logic based Ant Colony Optimization algorithm (FACO). The proposed algorithm takes into consideration the uncertainties that can be found in both the heuristic and the pheromone trails. This is achieved by representing the parameters of the problem and the metaheuristic algorithm as a pair of value and fuzzy level. The fuzzy level is considered as an indication of the uncertainty in the corresponding parameter. A stochastic-based technique is proposed to enable the artificial ant to choose the best incoming step based on the values of the probabilities and their corresponding fuzzy levels. The proposed FACO gives the optimal solution in a form of an optimal value and its corresponding fuzzy level. The proposed FACO is tested using the benchmark Quadratic Assignment Problem (QAP) and Travelling Salesman Problem (TSP). The results indicate that the developed FACO gives better optimal values with improved performance.

\section{Keywords:}

Ant Colony Algorithm, Quadratic Assignment Problem, Travelling Salesman Problem, Combinatorial Problems, Fuzzy Systems, Fuzzy-based Logic Algebra 


\section{Introduction:}

Combinatorial problems are intriguing because they are often easy to state but very difficult to solve. Some of the best known and widely applied metaheuristics are simulated annealing, tabu search, evolutionary computation,... etc have been developed to solve these problems. One of the important classes of combinatorial problems is QAP. Several methods such as particle swarm [1], genetic algorithm [2], tabu search [3] have been developed to solve these problems. Another class of the combinatorial problems is the class of TSP. Several methods such as genetic algorithm [4] have been developed to solve these problems. The world of metaheuristics is rich and multifaceted. Several characteristics make ACO a unique approach: it is a constructive, population-based metaheuristic which exploits an indirect form of memory of previous performance. This combination of characteristics is not found in any of other metaheuristics. Consequently, ACO techniques are one of the recent and most successful heuristic methods. Unfortunately, these algorithms have been developed for deterministic representation of the combinatorial problems. However, the practical systems, a need for developing efficient ACO technique that is able to represent all the uncertainties of the parameters of the system in a way suitable for performing calculations and giving optimal solution in reasonable time arises [5,6]. This paper introduces the development of such technique.

\section{Definition of proposed fuzzy logic-based arithmetic representation}

The algebra of fuzzy representations is based on a certain fuzzy level, given for the scalar forms. Let $\mathrm{X}$ is a general scalar parameter comprising two main components; as follows

$$
\mathrm{X}=\mathrm{X}_{0}+\mathrm{X}_{\mathrm{f}}
$$

where $X_{0}$ is the deterministic equivalence and $X_{f}$ is the fuzzy equivalence representing a small uncertainty or value tolerance in the parameter $\mathrm{X}$.

$\mathrm{X}=\mathrm{X}_{0}\left(1+\lambda_{\mathrm{X}}\right), \lambda_{\mathrm{X}}=\mathrm{X}_{\mathrm{f}} / \mathrm{X}_{0}$

The proposed fuzzy logic arithmetic representation is expressed by replacing each parameter with a pair of parentheses, the first is the actual value and the second is corresponding fuzzy level, that is (Value, Fuzzy Level). A summary of the main fuzzylogic based algebraic operations are presented in Table (1).

Table (1): Summary of most common fuzzy logic-based arithmetic algebra 


\begin{tabular}{|c|c|c|}
\hline $\begin{array}{l}\text { Multiplication and } \\
\text { Division }\end{array}$ & $\mathrm{X} . \mathrm{Y} / \mathrm{Z}$ & $\mathrm{X} . \mathrm{Y} / \mathrm{Z}=\left(\mathrm{X}_{0} \mathrm{Y}_{0} / \mathrm{Z}_{0}, \lambda_{\mathrm{X}}+\lambda_{\mathrm{Y}}-\lambda_{\mathrm{Z}}\right)$ \\
\hline $\begin{array}{l}\text { Addition } \\
\text { Subtraction }\end{array}$ & $\mathrm{X}-\mathrm{Y} \ldots+\mathrm{Z}$ & $\mathrm{W}_{0}=\mathrm{X}_{0}-\mathrm{Y}_{0} \ldots+\mathrm{Z}_{0}$, and $\lambda_{\mathrm{W}}=\frac{\left(\mathrm{X}_{0} \lambda_{\mathrm{X}}-\mathrm{Y}_{0} \lambda_{\mathrm{Y}} \ldots+\mathrm{Z}_{0} \lambda_{\mathrm{Z}}\right)}{\left(\mathrm{X}_{0}-\mathrm{Y}_{0} \ldots+\mathrm{Z}_{0}\right)}$ \\
\hline Other Polynomials & $\mathrm{X}^{\mathrm{m} / \mathrm{n}}$ & $\mathrm{Y}=\left(\mathrm{X}_{\mathrm{o}}^{\mathrm{n} / \mathrm{m}}, \lambda_{\mathrm{Y}}\right)$ and $\lambda_{\mathrm{Y}}=(\mathrm{n} / \mathrm{m}) \lambda_{\mathrm{x}}$ \\
\hline
\end{tabular}

\section{The developed fuzzy logic-based ACO algorithm}

Conventional ant colony algorithm uses the parameters of the problem, the heuristic information and the pheromone trails to solve the combinatorial optimization problems. Details for ACO can be found in [7]. In most of the practical problems, the parameters cannot be considered as crisp because they usually have different types of uncertainties. In this paper, a new FACO is presented to solve combinatorial problems with uncertainties to its parameters. These uncertainties should affect both the heuristic information and pheromone update factors of the algorithms. The uncertainties are represented as fuzzy levels. This means that heuristic information will be developed as follows.

The heuristic information is $\eta_{\mathrm{ij}}=1 /\left(\mathrm{d}_{0 \mathrm{ij}}, \lambda_{\mathrm{d}_{\mathrm{ij}}}\right)$

Applying the rules of fuzzy logic arithmetic representation, then

$\left(\eta_{0 \mathrm{ij}}, \lambda_{\eta_{\mathrm{ij}}}\right)=\left(1 / \mathrm{d}_{0 \mathrm{ij}},-\lambda_{\mathrm{d}_{\mathrm{ij}}}\right)$

where $\eta_{0 \mathrm{ij}}$ is the crisp value for heuristic information, $\lambda_{\eta_{\mathrm{ij}}}$ is the fuzzy level for $\eta_{0 \mathrm{ij}}, \mathrm{d}_{0 \mathrm{ij}}$ represents the crisp value for the weight between two nodes and $\lambda_{\mathrm{d}_{\mathrm{ij}}}$ represents the fuzzy level for $\mathrm{d}_{0 \mathrm{ij}}$.

When the tour is completed, the $K$ th ant lays down on the edge $(i, j)$ the pheromone value

$$
\left(\Delta \tau_{0 \mathrm{ij}, \mathrm{k}}(\mathrm{t}), \lambda_{\Delta \tau_{\mathrm{ij}, \mathrm{k}}(\mathrm{t})}\right)= \begin{cases}\left(\mathrm{Q} / \mathrm{L}_{0 \mathrm{k}}(\mathrm{t}),-\lambda_{\mathrm{L}_{\mathrm{k}}(\mathrm{t})}\right), & \text { if }(\mathrm{i}, \mathrm{j}) \in \mathrm{T}_{\mathrm{k}}(\mathrm{t}) \\ 0, & \text { if }(\mathrm{i}, \mathrm{j}) \notin \mathrm{T}_{\mathrm{k}}(\mathrm{t})\end{cases}
$$

where $\Delta \tau_{0 \mathrm{ij,k}}(\mathrm{t})$ is the crisp value of quantity of pheromone of the ant $\mathrm{k}$ at iteration $\mathrm{t}$, $\lambda_{\Delta \tau_{\mathrm{ij}, \mathrm{k}}(\mathrm{t})}$ is the fuzzy level for $\Delta \tau_{0 \mathrm{ij}, \mathrm{k}}(\mathrm{t}), \mathrm{L}_{0 \mathrm{k}}(\mathrm{t})$ is the crisp value for function (solution) of the ant $\mathrm{k}$ at iteration $\mathrm{t}, \lambda_{\mathrm{L}_{0 \mathrm{k}}{ }^{(t)}}$ is the fuzzy level for $\mathrm{L}_{0 \mathrm{k}}(\mathrm{t})$, and $\mathrm{Q}>0$ is an adjustable parameter. 
Thus, the crisp value of quantity of pheromone on the path is

$$
\Delta \tau_{0 \mathrm{ij}, \mathrm{k}}(\mathrm{t})=\Delta \tau_{\mathrm{ijj}, \mathrm{k}}(\mathrm{t})+\mathrm{Q} / \mathrm{L}_{\mathrm{Ok}}(\mathrm{t})
$$

Then, the fuzzy level for the quantity of pheromone on the path will be

$\lambda_{\Delta \tau_{\mathrm{ij}, \mathrm{k}}(\mathrm{t})}=\left(\Delta \tau_{0 \mathrm{ij}, \mathrm{k}}(\mathrm{t}) \lambda_{\Delta \tau_{\mathrm{ij}, \mathrm{k}}(\mathrm{t})}-\mathrm{Q} \lambda_{\mathrm{L}_{\mathrm{k}}(\mathrm{t})} / \mathrm{L}_{0 \mathrm{k}}(\mathrm{t})\right) /\left(\Delta \mathrm{t}_{0 \mathrm{ij}, \mathrm{k}}(\mathrm{t})+\mathrm{Q} / \mathrm{L}_{0 \mathrm{k}}(\mathrm{t})\right)$

Consequently, the crisp value for the pheromone update is

$\tau_{0 \mathrm{ij}}(\mathrm{t}+1)=(1-\rho) \tau_{0 \mathrm{ij}}(\mathrm{t})+\Delta \tau_{0 \mathrm{ijj}, \mathrm{k}}(\mathrm{t})$

The fuzzy level for the pheromone update equation is

$\lambda_{\tau_{\mathrm{ij}}(\mathrm{t}+1)}=\left((1-\rho) \tau_{0 \mathrm{ij}}(\mathrm{t}) \lambda_{\tau_{\mathrm{ijj}}(\mathrm{t})}+\Delta \tau_{0 \mathrm{ij}, \mathrm{k}}(\mathrm{t}) \lambda_{\Delta \tau_{\mathrm{ij}, \mathrm{k}}(\mathrm{t})}\right) /\left((1-\rho) \tau_{0 \mathrm{ij}}(\mathrm{t})+\Delta \tau_{0 \mathrm{ij}, \mathrm{k}}(\mathrm{t})\right)$

In the conventional ACO algorithm, each ant uses the pheromone trail to calculate the probability. Then the ant chooses the node with higher probability.

In the proposed technique, each probability has its own fuzzy level. Now, the ant has a problem of making a decision in using fuzzy probabilities. Fuzzy probabilities mean that when the ant calculates the probabilities of choosing node $x_{n}$ as depicted in Figure (1), this value has a corresponding fuzzy level according to (11).

Consequently, the crisp value for probability equation is

$\mathrm{P}_{0 \mathrm{ij}}^{\mathrm{k}}(\mathrm{t})=\left[\tau_{0 \mathrm{ij}}(\mathrm{t})\right]^{\alpha}\left[\eta_{0 \mathrm{ij}}\right]^{\beta /} / \sum_{1 \in J_{\mathrm{ik}}}\left[\tau_{0 \mathrm{ij}}(\mathrm{t})\right]^{\alpha}\left[\eta_{0 \mathrm{ijj}}\right]^{\beta}$

Then the fuzzy level for the probability equation is

$\lambda_{\mathrm{p}_{\mathrm{ij}}^{\mathrm{k}}(\mathrm{t})}=\left(\left(\left[\alpha \lambda_{\tau_{\mathrm{ij}}(\mathrm{t})}\right]+\left[\beta \lambda_{\left.\eta_{\mathrm{ijj}}\right]}\right)-\frac{\sum_{l \in J_{i \mathrm{i}}}\left(\left[\alpha \lambda_{\tau_{\mathrm{ij}}(\mathrm{t})}\right]+\left[\beta \lambda_{\eta_{\mathrm{ij}}}\right]\right)\left[\tau_{0 \mathrm{ij}}(\mathrm{t})\right]^{\alpha}\left[\eta_{0 \mathrm{ij}}\right]^{\beta}}{\sum_{l \in J_{i k}}\left[\tau_{0 \mathrm{ij}}(\mathrm{t})\right]^{\alpha}\left[\eta_{0 \mathrm{ij}}\right]^{\beta}}\right)\right.$.

The decision making algorithm under these fuzzy conditions is based on assuming that each of the calculated probabilities has a PDF of certain type spread between $\mathrm{P}_{0}$ and $\mathrm{P}_{0}\left(1+\lambda_{\mathrm{P}}\right)$. Then, the decision of the ant will be based on the average of the PDF. The PDF may be Gaussian, Beta, linear, nonlinear,...etc. However, in this approach, the linear probability density function can be considered. 

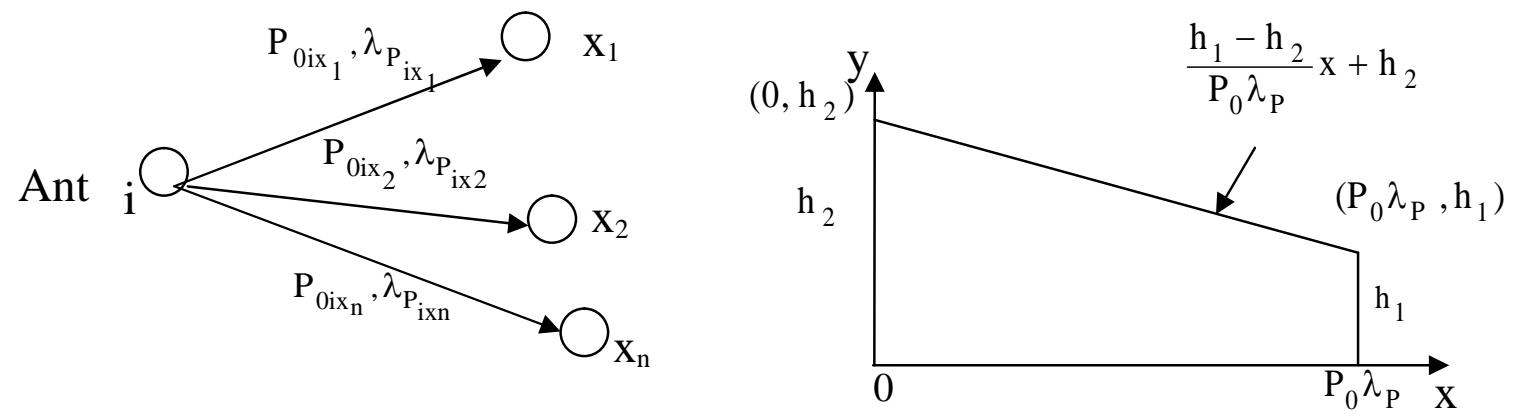

Figure (1): The probability between the node and the other nodes.

Figure (2): The probability density function of system fuzziness is trapezoidal.

The PDF of system fuzziness is assumed to be trapezoidal as depicted in Figure (2). It is defined in the interval $\left[0, \mathrm{P}_{0} \lambda_{\mathrm{P}}\right]$. The relationship between $\mathrm{h}_{1}, \mathrm{~h}_{2}, \mathrm{P}_{0}$, and $\lambda_{\mathrm{P}}$ can be given by (12)

$\frac{\left(\mathrm{h}_{1}+\mathrm{h}_{2}\right)}{2} \cdot \mathrm{P}_{0} \lambda_{\mathrm{P}}=1$,

This is a direct result as the area under the PDF should equal to 1.

The equation of the inclined line is given by $y=\frac{\mathrm{h}_{1}-\mathrm{h}_{2}}{\mathrm{P}_{0} \lambda_{\mathrm{P}}} \mathrm{x}+\mathrm{h}_{2}$

Using the above equation, the average value can be calculated as follows.

$$
\begin{aligned}
\text { Average value } & =\int_{0}^{\lambda_{\mathrm{P}} \mathrm{P}_{0}}\left(\frac{\mathrm{h}_{1}-\mathrm{h}_{2}}{\mathrm{P}_{0} \lambda_{\mathrm{P}}} \mathrm{x}+\mathrm{h}_{2}\right) \mathrm{xdx}, 0 \leq \mathrm{x}<\lambda_{\mathrm{P}} \mathrm{P}_{0} \\
& =\frac{2 \mathrm{P}_{0} \lambda_{\mathrm{P}}}{6}+\mathrm{h}_{1} \frac{\mathrm{P}_{0}^{2} \lambda_{\mathrm{P}}^{2}}{6}
\end{aligned}
$$

The special cases of (15) are given below:-

1) For $h_{1}=h_{2}$, the PDF of the system fuzziness is uniform. Then, the average value is given by $\mathrm{P}_{0}\left(1+\lambda \lambda_{\mathrm{P}}\right)$, where $\lambda=1 / 2$.

2) For $h_{1}=0$, and from (12), $h_{2}=2 / P_{0} \lambda_{P}$, the PDF of the system fuzziness is triangle with negative slope and consequently the probability between two nodes that is used in the decision making $=\mathrm{P}_{0}\left(1+\lambda \lambda_{\mathrm{P}}\right)$ where $\lambda=1 / 3$.

3) For $_{2}=0$, and from (12), $h_{1}=2 / P_{0} \lambda_{P}$, the PDF of the system fuzziness is triangle with positive slope and consequently the probability between two nodes that is used in 
the decision making $=\mathrm{P}_{0}\left(1+\lambda \lambda_{\mathrm{P}}\right)$ where $\lambda=2 / 3$.

The above discussion reveals that the probability between two nodes is given by $\mathrm{P}_{0}\left(1+\lambda \lambda_{\mathrm{P}}\right)$, where $\lambda \in[1 / 3,2 / 3]$.

From the previous arguments, it is to be noted that when the PDF is assumed to be linear with single and/or double rules, the average value that can be used by the ant to determine the next node has the range one third to two thirds of the fuzzy level. Thus, the general form of the probabilities that is as follows.

$$
\mathrm{P}_{0 \mathrm{ij}}^{\mathrm{k}}(\mathrm{t})=\mathrm{P}_{\mathrm{ij}}^{\mathrm{k}}(\mathrm{t})\left(1+\lambda \lambda_{\mathrm{P}_{\mathrm{ij}}^{\mathrm{k}}(\mathrm{t})}\right) \quad 0 \leq \mathrm{P}_{\mathrm{ij}}^{\mathrm{k}}(\mathrm{t}) \leq 1
$$

where $\lambda \in[1 / 3,2 / 3]$

\section{The application of the developed fuzzy logic-based ant colony optimization to TSP}

The TSP can be stated as the problem of finding a minimal time required to go through a tour with constraints that visits at each town must be once. This problem with uncertainties to its parameters can be solved by applying the developed FACO.

During the construction of the solution, the decision of an ant to travel from its current position to its next position depends on the proposed technique. That is to say, the probabilities of going from the current position to its neighbours are calculated with their fuzzy levels. Then, the averages are calculated using (16) with the given value of $\lambda$. The next position of the ant is the position that has maximum average.

At the end of iteration, the pheromone trails and their corresponding fuzzy levels are updated. This is done in two steps. The first is the pheromone deposited as given in (6) and its corresponding fuzzy level according to (7). The second is the pheromone evaporation according to (8) and its corresponding fuzzy level according to (9). The iterations continue until stopping criterion is achieved. The tour time of each ant and its corresponding fuzzy level are calculated. Then, the minimum tour time is the best of this iteration. The optimal tour time is the minimum of the best tour time of the current iteration and the minimum tour time of all iterations. The path which gives the tour time is given in (17) and its corresponding fuzzy level is given in (18).

The total time $\mathrm{L}_{0 \mathrm{k}}(\mathrm{t})=\sum_{\mathrm{i}=1}^{\mathrm{n}-1} \mathrm{~d}_{\pi(\mathrm{i}) \pi(\mathrm{i}+1)}+\mathrm{d}_{\pi(\mathrm{n}) \pi(1)}$

The fuzzy level for the total time $\lambda_{\mathrm{L}_{\mathrm{k}}(\mathrm{t})}=\frac{\sum_{\mathrm{i}=1}^{\mathrm{n}-1} \mathrm{~d}_{\pi(\mathrm{i}) \pi(\mathrm{i}+1)} \lambda_{\mathrm{d}_{\pi(\mathrm{i}) \pi(\mathrm{i}+1)}}+\mathrm{d}_{\pi(\mathrm{n}) \pi(1)} \lambda_{\mathrm{d}_{\pi(\mathrm{n}) \pi(1)}}}{\sum_{\mathrm{i}=1}^{\mathrm{n}-1} \mathrm{~d}_{\pi(\mathrm{i}) \pi(\mathrm{i}+1)}+\mathrm{d}_{\pi(\mathrm{n}) \pi(1)}}$ 
The parameters of the algorithm are chosen to be alpha $=1$, beta $=5$, number of ants $=30$, rho $=0.1$ and $\mathrm{Q}=100$. In addition, the parameter $\lambda$ should discussed in the previous section is changed from 0 to 1 . The Oliver30 benchmark TSP which has 30 cities and described in [8] is used. The best time and the average time when $\lambda=0$, at each iteration, are shown in Figure (3). From the Figure, the time of the shortest tour is $423.5129 \mathrm{~min}$ and its corresponding fuzzy level equals to $0.038 \mathrm{~min}$.

In order to complete the results, $\lambda$ should take values other than zero. As discussed in the above section, When this value of $\lambda$ is $1 / 3$, the proposed algorithm gives the results shown in Figure (4). As depicted in the Figure, the solution is improved to 420.0115 min with corresponding fuzzy level equals to $0.6797 \mathrm{~min}$. The Figure also shows that the response of the solution also is improved. That is to say, the optimal solution is reached in a relatively small number of iterations. Also, the average time at each iteration has fewer fluctuations than the previous case which is shown in Figure (3).

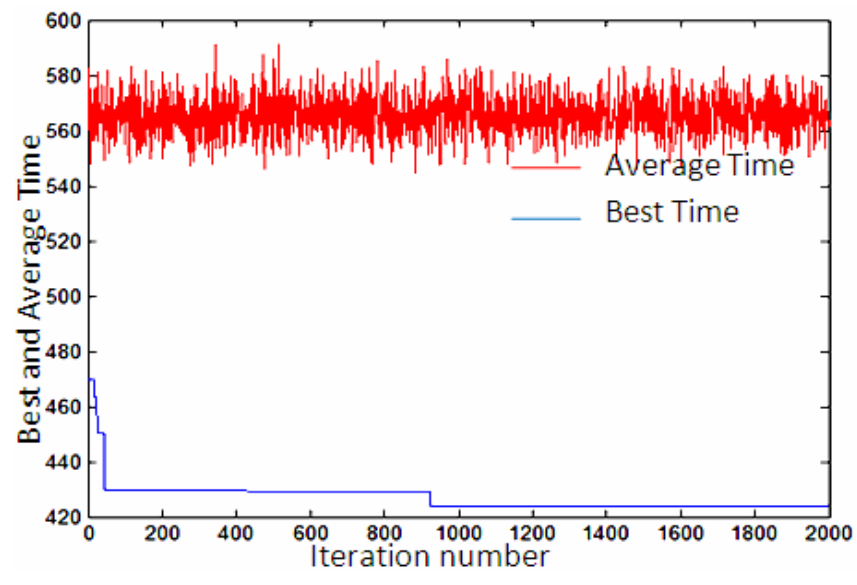

Figure (3): Best and average time at each iteration, where $\lambda=0$

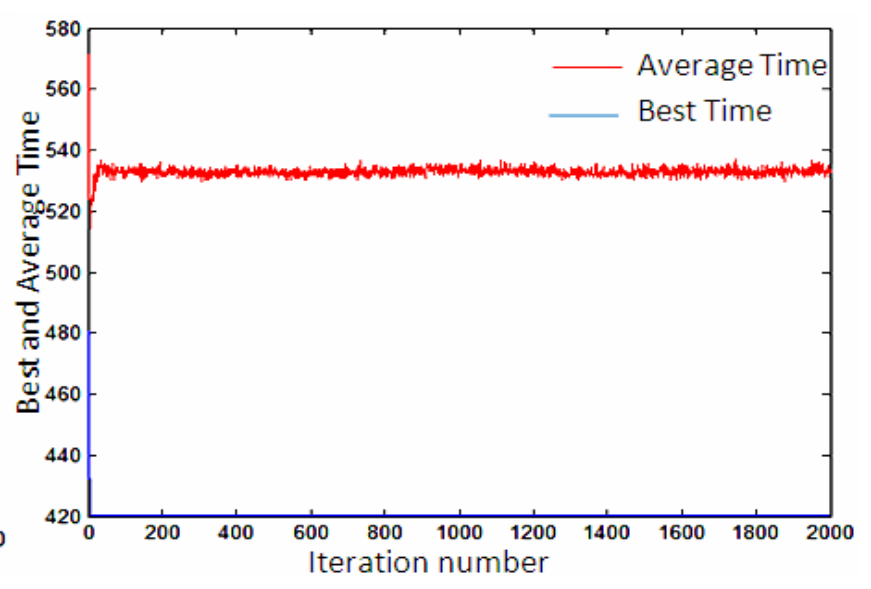

Figure (4): Best and average time at each iteration where $\lambda=0.333$

When the value of $\lambda$ is $1 / 2$, the proposed algorithm gives the results shown in Figure (5). As depicted in the Figure, the solution is improved to $419.288 \mathrm{~min}$ with corresponding fuzzy level equals to $.505 \mathrm{~min}$.

When the value of $\lambda$ is $2 / 3$, the proposed algorithm gives the results shown in Figure (6). As depicted in the Figure, the solution is improved to 418.488 min with corresponding fuzzy level equals to $1.216 \mathrm{~min}$. Also, the response of the solution is improved and the fluctuations of the average time at each iteration are decreased.

Table (2) summarize the effect of the value of $\lambda$ on the solution of TSP with uncertanity to its parameters. 


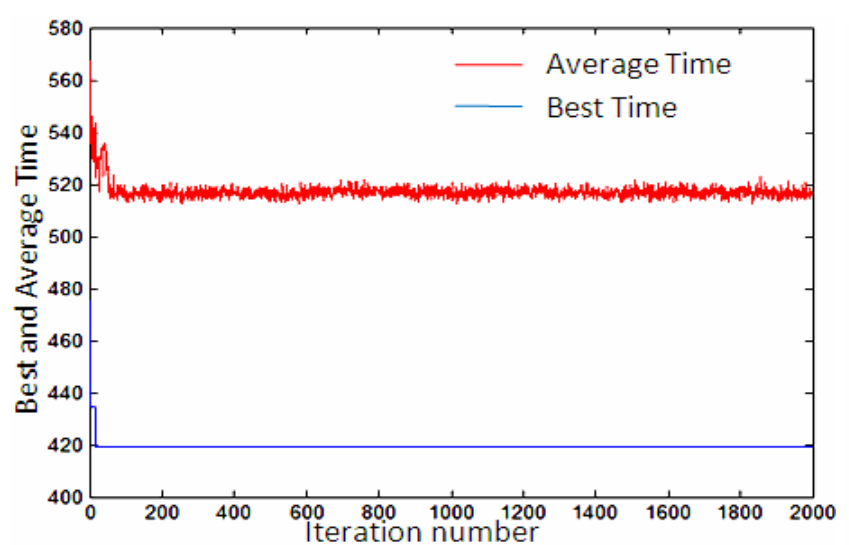

Figure (5): Best and average time at each iteration where $\lambda=0.5$

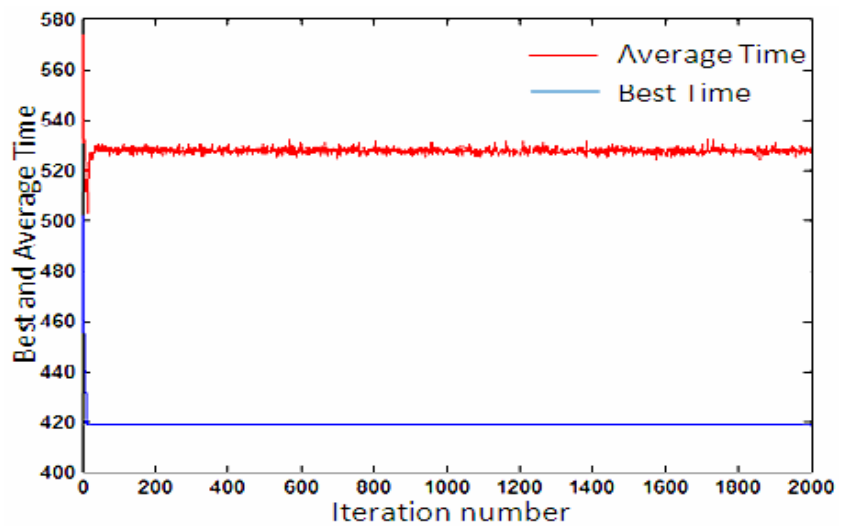

Figure (6): Best and average time at each iteration where $\lambda=0.667$

Table (2): Effect of FACO on the solution of TSP with uncertainties to its parameters

\begin{tabular}{|c|c|c|c|c|c|c|}
\hline$\lambda$ & $\begin{array}{c}\text { ACO } \\
\lambda=\mathbf{0}\end{array}$ & $\begin{array}{c}\text { FACO } \\
\lambda=\mathbf{0 . 3 3 3}\end{array}$ & $\begin{array}{c}\text { FACO } \\
\lambda=\mathbf{0 . 5}\end{array}$ & $\begin{array}{c}\text { FACO } \\
\lambda=\mathbf{0 . 6 6 7}\end{array}$ & $\begin{array}{c}\text { FACO } \\
\lambda=\mathbf{0 . 8}\end{array}$ & $\begin{array}{c}\text { FACO } \\
\lambda=\mathbf{1}\end{array}$ \\
\hline Value (min) & 423.741 & 420.0115 & 419.288 & 418.488 & 425.532 & 427.514 \\
\hline Fuzzy Level & 0.0375 & 0.6797 & 0.505 & 1.216 & 0.635 & 1.443 \\
\hline
\end{tabular}

It is to be noted that the above results are better than those in [9], where genetic algorithms were applied to solve the Oliver30 problem; they could find a tour of length $424.635 \mathrm{~min}$. The same result was often obtained by ant-cycle [10], which also found a tour of length $423.741 \mathrm{~min}$.

\section{The developed fuzzy logic-based ant colony optimization system for QAP}

To apply the FACO metaheuristic to assignment problems, a first step is to map the problem on a construction $\operatorname{graph}_{\mathrm{G}_{\mathrm{C}}}=(\mathrm{C}, \mathrm{L})$, where $\mathrm{C}$ is the set of components (usually the components consists of all locations and all the facilities) and $\mathrm{L}$ is the set of connections that fully connects the graph. Transitions are from facilities to locations and vice versa. Typically, an ant first chooses facility, then a location to which to assign the facility, then another facility, and so forth, until all facilities have been assigned. Facilities and locations are chosen from the feasible neighbouhood, that is, from facilities (locations) not signed yet. These constraints can be easily enforced in the ants' walk by building only coupling between still unsigned facilities and locations.

$$
f(\pi)=\sum_{i=1}^{n} \sum_{j=1}^{n} b_{i j} a_{\pi_{i} \pi_{j}}
$$




$$
\lambda_{f(\pi)}=\sum_{i=1 j=1}^{n} \sum_{i j}^{n} b_{\pi_{i} \pi_{j}}\left(\lambda_{b_{i j}}+\lambda_{a_{\pi_{i} \cdot \pi j}}\right) / \sum_{i=1}^{n} \sum_{j=1}^{n} b_{i j} a_{\pi_{i} \pi_{j}}
$$

where $a_{i j}$ is the distance between locations $i$ and $j, \pi_{i}$ gives the location of facility $i$ in the current solution $\pi \in S$ ( $n$ and $S$ is the candidate solution. The term $b_{i j} a_{\pi_{1} \pi_{j}}$ describes the cost contributions of simultaneously assigning facility $i$ to location $\pi_{1}$ and facility $j$ to location $\pi_{\mathrm{j}}$ and $\left(\lambda_{\mathrm{b}_{\mathrm{ij}}}+\lambda_{\mathrm{a}_{\mathrm{i}} \pi_{\mathrm{j}}}\right)$ represents the fuzzy level for $\mathrm{b}_{\mathrm{ij}} \mathrm{a}_{\pi_{\mathrm{i}} \pi_{\mathrm{j}}}$.

This problem with uncertainties to its parameters can be solved by applying the developed FACO algorithm. The input parameters are the time between locations, the flow between facilities and the parameters of ACO. Each time and each flow is then assigned a fuzzy level.

In this case, a real assignment problem with large size is taken into consideration. The problem is the optimum allocation of services in the offices of a multinational company located in Milan, Italy, as described in [11].The size of the problem is 33.

Table (3): Effect of FACO on the solution of QAP with uncertainties to its parameters

\begin{tabular}{|c|c|c|c|c|c|c|}
\hline$\lambda$ & $\begin{array}{c}\text { ACO } \\
\lambda=\mathbf{0}\end{array}$ & $\begin{array}{c}\text { FACO } \\
\lambda=\mathbf{0 . 3 3 3}\end{array}$ & $\begin{array}{c}\text { FACO } \\
\lambda=\mathbf{0 . 5}\end{array}$ & $\begin{array}{c}\text { FACO } \\
\boldsymbol{\lambda}=\mathbf{0 . 6 6 7}\end{array}$ & $\begin{array}{c}\text { FACO } \\
\boldsymbol{\lambda}=\mathbf{0 . 8}\end{array}$ & $\begin{array}{c}\text { FACO } \\
\boldsymbol{\lambda}=\mathbf{1}\end{array}$ \\
\hline $\begin{array}{c}\text { Value(man-second } \\
\text { per week) }\end{array}$ & 438786 & 433506 & 432614 & 430946 & 440730 & 442212 \\
\hline Fuzzy Level & 2.009 & 2.485 & 2.4817 & 2.3837 & 2.6135 & 2.301 \\
\hline
\end{tabular}

The above results are better than those found in [11], where ACO were applied to solve this problem; the value of the objective function is 438786 man-seconds per week. The problem is then solved using different values of $\lambda$. The results are shown in Table (3). It is noted that the proposed algorithm gives better values for TSP and QAP, than those given by the conventional ACO. Furthermore, the range of the parameter $\lambda$ that gives better values is $(1 / 3 \leq \lambda \leq 2 / 3)$. Beyond this range, the solution deteriorates.

Taking $\lambda$ greater that $2 / 3$ means that the assumed probability density function covers a range from $\mathrm{P}$ to greater than $\mathrm{P}_{0}\left(1+\lambda \lambda_{\mathrm{P}}\right)$, but according to the definition of the fuzzy level, a probability of value $P$ and a fuzzy level of $\lambda_{P}$ cannot take values beyond $\mathrm{P}_{0}\left(1+\lambda \lambda_{\mathrm{P}}\right)$. Therefore, any probability density function that covers range from $\mathrm{P}$ to greater than $\mathrm{P}_{0}\left(1+\lambda \lambda_{\mathrm{P}}\right)$ is not reasonable. 


\section{Conclusions:}

A new FACO technique has been developed. The main advantage of the proposed technique is its ability to represent the uncertainties of the parameters of both the optimization problem and the meta-heuristic algorithm in a fuzzy logic-based form. Consequently, the proposed FACO has the ability to give the optimal solution in a form of an optimal value and its corresponding fuzzy level. The fuzzy level of the solution is shown to be interpreted as the uncertainty in the value of the optimal solution. The proposed technique has been tested using two classes of combinatorial problems. The results have been compared to other techniques found in the literature. This comparison indicates that the developed FACO gives better optimal values. Furthermore, the proposed technique achieves the optimal solution in number of trials less than those required by the conventional techniques. This means that the proposed technique has improved the quality of the solution and decreased its time. 


\section{References:}

[1] Liu H., Abraham A., and Zhang J., "A Particle Swarm Approach to Quadratic Assignment Problems", Soft Computing in Industrial Applications, ASC 39, pp. 213-222, 2007.

[2] Liong Choong Yeun, Wan Rosmanira Ismail and Mourad Zirour, "Application of Genetic Algorithm in a Special Quadratic Assignment Problem", Preceding of the 2nd IMT-GT Regional Conference on Mathematics, Statistics and Applications Universities Sains Malaysia, Penang, pp.13-15, June 2006.

[3] Tabitha James, César Rego, and Fred Glover, "Multistart Tabu Search and Diversification Strategies for the Quadratic Assignment Problem", IEEE Transactions On Systems, Man, And Cybernetics—Part A: Systems And Humans, Vol. 39, No. 3, May 2009.

[4] M. Dorigo, V. Maniezzo, and A. Colorni, "Ant system: optimization by a colony of cooperating agents," IEEE Transaction on Systems, Man, and Cybernetics, Part B., vol. 26, no. 1, pp. 29-41, 1996.

[5] Kandasamy, W. B. Vasantha, Smarandache, Florentin, and Ilanthenral K.,Elementary Fuzzy Matrix Theory and Fuzzy Models for Social Scientists. Automation, Los Angeles, USA, 2007.

[6] Walaa Ibrahim Gabr and Hassen Taher Dorrah, "New Fuzzy Logic-based Arithmetic and Visual Representations for Systems' Modelling and Optimization" IEEE International Conference on Robotics and Biomimetics, Bangkok, Thailand., Paper No. 8 , December $14-17,2008$.

[7] Dorigo Marco and Stutzle Thomas, Ant Colony Optimization Bradford Book , 2004.

[8] TSPLIB: A Traveling Salesman Problem Library. http://www.iwr.uni heidelberg.de/groups/comopt/ software/TSPLIB95/.

[9] Whitley D., Starkweather T., Fuquay D., "Scheduling Problems and Travelling Salesman: the Genetic Edge Recombination Operator," Proceedings of the Third International Conference on Genetic Algorithms, Morgan Kaufmann, 1989.

[10] M. Dorigo, V. Maniezzo, and A. Colorni, "Ant system: optimization by a colony of cooperating agents," IEEE Transaction on Systems, Man, and Cybernetics, Part B., vol. 26, no. 1, pp. 29-41, 1996.

[11] Vittorio Maniezzo and Alberto Colorni, "The Ant System Applied to the Quadratic Assignment Problem", IEEE Transactions on Knowledge and Data Engineering, Vol. 11, No. 5, September/October 1999. 